Annales Geophysicae (2002) 20: 1529-1538 (C) European Geosciences Union 2002

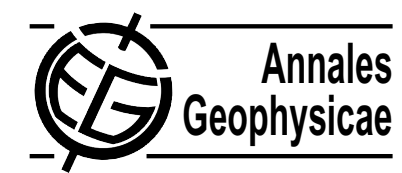

\title{
Actively produced high-energy electron bursts within the magnetosphere: the APEX project
}

\author{
L. Přech, Z. Němeček, J. Šafránková, and A. Omar \\ Charles University, Faculty of Mathematics and Physics, V Holesovickach 2, 18000 Praha 8, Czech Republic
}

Received: 25 June 2001 - Revised: 15 March 2002 - Accepted: 17 April 2002

\begin{abstract}
The APEX project (Active Plasma Experiment) has been launched into a polar orbit in December 1991 and consists of two satellites (IK-25 and MAGION-3), with a distance between them from $200 \mathrm{~km}$ to $10000 \mathrm{~km}$. The mission used intensive electron beam emission, complemented by a low-energy Xenon plasma generator during the electron beam injection, for the study of dynamic processes in the magnetosphere and upper ionosphere.

The paper deals with short, intensive bursts of fieldaligned electrons observed during the APEX mission on board the MAGION-3 satellite. These events are located predominantly at the middle geomagnetic latitudes in the dayside magnetosphere. The time-energy structure of these electron bursts is similar to the inverted-V one, but the pitchangle width is less than $10^{\circ}$. Electrons with an energy up to $700 \mathrm{keV}$ are often observed during the events. We analyze the observed events, discuss the possible mechanisms of the particle spreading, and the role of the main satellite's activity as a possible source of these events.
\end{abstract}

Key words. Ionosphere (particle acceleration; particle precipitation) - Space plasma physics (active perturbation experiments)

\section{Introduction}

1.1 Observations of electron beams in the magnetosphere

The energy-time signatures of particle fluxes measured in the magnetosphere can often be used to identify and characterize distant sources. The isolated field-aligned electron bursts are observed, for example, in the boundary plasma sheet region of the auroral oval (Johnstone and Winningham, 1982). The characteristic properties of these fluxes are a maximum energy of about $1 \mathrm{keV}$, a duration of $\sim 10 \mathrm{~s}$, a broad energy

Correspondence to: L. Přech

(lubomir.prech@mff.cuni.cz) spectrum, and pitch angles less than $30^{\circ}$. Torbert and Carlson (1980) reported similar events for the dayside auroral oval, again in the discrete aurora region. In the nightside part of the diffuse aurora region, Clemmons et al. (1994) found high-energy electron bursts, which lasted $\sim 2 \mathrm{~s}$ and extended to energies of a few $\mathrm{keV}$, presumably of plasma sheet or ring current origin. The authors calculated a source location only about $850 \mathrm{~km}$ above the spacecraft for one of two examples, and for the second event, the source was inferred to lie near the equatorial region in the early morning sector at a distance of about $6.5 \mathrm{R}_{\mathrm{E}}$ from the spacecraft.

The data set obtained from the FREJA mission includes many examples of such events. One of them, inverted-V electrons, have been reported in Haerendel et al. (1994) during some passes over broad auroral arc systems. A combination of these observations of inverted- $\mathrm{V}$ events and groundbased optical measurements leads the authors to the conclusion that the fine structure of the observed events seems to originate from modulations of the accelerating field-aligned potential probably at the interface between the topside ionosphere and hot magnetospheric plasma. Boehm et al. (1994) have reported a high-resolution measurement of very narrow $(0.5 \div 5 \mathrm{~km})$ inverted-V structures observed on the edge of the inverted-V region (McFadden et al., 1986; McFadden et al., 1990). These precipitations have been analyzed and two possible mechanisms for the particle acceleration were proposed: the Alfvén ion-cyclotron waves or the field-aligned potential drop (McFadden et al., 1986; Temerin et al., 1986). These two mechanisms are supposed to cause the acceleration of the cold ionospheric electrons to the observed energies (up to $25 \mathrm{keV}$ ). Nevertheless, neither mechanism can explain all the features of these events; the Alfvén wave acceleration theories cannot easily explain why the field-aligned electron energy extends up to, but not beyond, the energy of the isotropic inverted- $\mathrm{V}$ populations, and the theories involving the $\mathrm{V}$-shape potential have trouble explaining how the cold ionospheric electrons enter the acceleration region (McFadden et al., 1986; Lotko, 1986).

On the other hand, electrons with higher energies were 
observed in different regions in low and middle altitudes. Microbursts of precipitating electrons, which were first described by Anderson and Milton (1964), were detected from balloons and their energies were mostly in the range of tens to hundreds of $\mathrm{keV}$. The microbursts were characterized by durations of $\sim 0.2 \mathrm{~s}$ and interburst spacings of $\sim 0.6 \mathrm{~s}$. Most observations of microbursts have involved measurements of the bremsstrahlung X-rays produced by the electrons as they encounter the atmosphere (e.g. Bering et al., 1988).

One set of early observations of microbursts was made from the INJUN 3 satellite and involved direct measurements of precipitating electrons with energies of $>40 \mathrm{keV}$ (Oliven et al., 1968). These microbursts were found to occur predominantly at $L=6 \div 8.5$ and MLT from 04:30 to 12:30 hours. Short bursts of electrons with the same energetic range (above $45 \mathrm{keV}$ ) were studied with data from the S81-1 lowaltitude polar-orbiting satellite (Imhof et al., 1989). The observed bursts met all of the criteria for microbursts and had soft spectra with negligible flux above $1 \mathrm{MeV}$.

Later papers present the observations of relativistic electron microbursts with durations of $>1 \mathrm{~s}$ (Imhof et al., 1991) and $<1$ s (Imhof et al., 1992). However, these relativistic electron microbursts occurred much more often near local midnight than near local noon. This local time preference of relativistic microbursts is opposite to the published local time occurrence of lower-energy electron microbursts (Oliven et al., 1968; Imhof et al., 1989).

Some relativistic electron precipitation bursts, many of which were observed in the drift loss cone, were previously found to occur in the vicinity of the plasmapause position estimated from the $K_{P}$ index (Imhof et al., 1986). These bursts were predominantly in the $L$ shell range of $\sim 4$ to $\sim 7$ and, therefore, probably near the trapping boundary. Many of these events were found very close to the outer boundary of the radiation belt measured on the same satellite pass. Enhanced precipitation is known to occur often near the last closed field line. From a low-altitude polar-orbiting satellite, the precipitation at this location displays $L$-dependent energy selectivity on the time scale of a few seconds or a distance of a few kilometers (e.g. Imhof et al., 1988; Imhof et al., 1991). The precipitating mechanism may be due to the loss of adiabatic motion when the radius of field line curvature is not significantly greater than the gyroradius of the electrons (e.g. Popielawska et al., 1985; Büchner and Zelenyi, 1989; Imhof et al., 1997).

All described events have been observed by numerous space missions including the APEX (Active Plasma EXperiment) project (e.g. Němeček et al., 1997) at high latitudes. However, the discussed bursts of energetic electrons have been recorded at middle and low geomagnetic latitudes during the APEX mission. The energy of these electrons extends up to several hundreds of $\mathrm{keV}$. One of the interesting features of these bursts is that, in spite of the small distance (from 10 to $1000 \mathrm{~km}$ ) between the main satellite, IK-25, and its subsatellite, MAGION-3, the bursts have been observed always by the subsatellite only. This fact suggests that the origin of these bursts is connected with the proximity of the main satellite and with the emission of the high-energy electrons and/or ions from the guns aboard of the main satellite. For this reason, we are giving a brief summary of results of active experiments in the next section.

\subsection{Active experiments}

Numerous experiments involving the injection of electron beams into space plasma have been carried out with the motivation to study the response of the space environment to controlled disturbances. The common feature of this simulation is an intense flux of suprathermal electrons created around such a rocket or satellite. For example, the Polar 5 motherdaughter payload configuration determined the dimension of the hot electron cloud transverse to $\boldsymbol{B}$ to be several beam Larmor radii and the dimension of the cloud along $\boldsymbol{B}$ to be about $100 \mathrm{~m}$ (Jacobsen, 1982). These dimensions are similar to those reported from a joint US/Canadian electron accelerator experiment (Duprat et al., 1983). The Echo 6 flight (Winckler et al., 1984) with a higher beam power showed a disturbed plasma region greater than $140 \mathrm{~m}$ of radius. Large disturbed regions of radius up to $800 \mathrm{~m}$ were reported by Sagdeev et al. (1981) in a lower altitude region. A very significant result from the Polar 5 and Echo 3 observations of suprathermal electrons is that the intensity of this population increases with altitude (Maehlum et al., 1980; Arnoldy and Winckler, 1981).

New results have been summarized by Hastings (1995) who reviewed plasma interactions with spacecraft in lowEarth orbit. His paper includes charging on polar orbits, ram and wake flows, use of high-voltage power systems in space, noise generation in self-induced plasma clouds around a large, active spacecraft, especially the Shuttle, anomalous ionization of emitted neutral gases, use of electrodynamic tethers and plasma contactors, and phenomena associated with the use of electrically propelled rockets.

Interesting and important results related to our topic showing the particle acceleration were obtained through Tethered Satellite Missions. During the operation of the Fast Pulsed Electron Gun (FPEG) (TSS-1 mission), the SPREE electrostatic analyzers measured intense fluxes of electrons at energies up to the energy of the emitted beam (100 mA, $1 \mathrm{keV}$ ). At energies above the charging peak, the shape of the electron distribution function was consistent with a simple acceleration by the electric field produced by the positively charged Orbiter (Oberhardt et al., 1993). Particle measurements up to $27 \mathrm{keV}$ were performed on the TSS-1R to track the spacecraft potential and collected current. In addition to the accelerated ionospheric electrons $(\sim 70 \mathrm{eV})$, a suprathermal population of electrons was observed to be centered around $200 \mathrm{eV}$ (Winningham et al., 1998). The authors assumed an AC acceleration of the suprathermal electrons whose free energy is gained from the differential $(\boldsymbol{E} \times \boldsymbol{B}$ driven) drift between electrons and ions.

Hardy et al. (1995) reported the measurements by the Shuttle Potential and Return Electron Experiment (SPREE), acquired during a period of the Electrodynamic Tethered 
Satellite mission when the fast pulsed electron generator injected a $1 \mathrm{keV}$ electron beam nearly perpendicular to the Earth's magnetic field. They detected part of the beam returning after the electrons executed almost a full gyrocycle. The electron spectrum was strongly peaked at $1 \mathrm{keV}$, but electrons with energies several hundred $\mathrm{eV}$ above (up to $1850 \mathrm{~V}$ ) and below the emitted beam energy were observed. It means that the beam energy was nearly doubled during one gyrorevolution.

The response of the ambient ionospheric plasma to the emission of the intensive $(8 \mathrm{keV}, 100 \mathrm{~mA})$ electron beam was studied by Němeček et al. (1997). The authors showed that the electron gun firing creates a disturbance that produces a broad spectrum of energetic electrons which extends up to $1500 \mathrm{eV}$. The acceleration process can be explained when an electric field with intensity of about $100 \mathrm{~V} / \mathrm{m}$ is expected. This intensity was in agreement with the observed $\boldsymbol{E} \times \boldsymbol{B}$ drift velocity.

The disadvantage of most of these experiments is that the response was studied in the close surrounding of emitting rockets (or satellites). Therefore, the APEX project, with two satellites separated by a relatively long distance, can bring qualitative new information on the propagation of the artificial disturbances in the magnetosphere.

As it was noted above, the observations of the field-aligned electron bursts on board the MAGION-3 satellite in low latitudes have been discussed first time by Němeček et al. (1996) based on the measurements of a low-energy electron spectrometer. The present paper brings new observational facts on this phenomenon and continues the discussion on its possible source from the point of view of the role of the active experiments carried out on board the main satellite.

\section{Instrumentation}

The APEX mother-daughter project was launched on 18 December 1991 and its two satellites moved along the same orbit with apogee $3080 \mathrm{~km}$, perigee $450 \mathrm{~km}$, and inclination $82.5^{\circ}$. The main satellite (IK-25) had a stabilized three-axis orientation with the axis of nearly axisymmetrical shape of the satellite oriented along the gradient of the gravitational field. The main axis of the small subsatellite (MAGION-3) was oriented parallel to the magnetic field.

The IK-25 satellite carried the electron gun that provided controlled injection of the electron beam. In all cases reported in the present study, the electron beam was injected in the direction opposite to the vector of the satellite's velocity. This resulted in a smooth change of the beam's pitch angle in time (or along the orbit) with a slope of $4^{\circ}$ per minute in the middle latitudes. The basic cycle of electron injection lasted about $23 \mathrm{~s}$ and was formed by current pulses of duration either 2 or $32 \mathrm{~ms}$. The pulses were repeated with a frequency swept in the range either $30 \mathrm{~Hz} \div 250 \mathrm{kHz}$ or $30 \mathrm{~Hz} \div 16 \mathrm{kHz}$. During this cycle, the gun energy was approximately $10 \mathrm{keV}$ and its mean current varied from 0.01 to $0.125 \mathrm{~A}$ (Dokukin, 1992).
The electron beam emission was complemented by a lowenergy Xe plasma releaser. This plasma injector was working in several different modes: the neutral Xe cloud release, the release of ionized Xe plasma with/without modulation, etc. The amount of released Xe was about $3 \mathrm{mg} / \mathrm{s}$. The release of neutral Xe started usually $\sim 35 \mathrm{~s}$ before the electron emission and the ionizer began to work $5 \mathrm{~s}$ before the electron emission. The ionizer produced about $10^{19}$ ions/s into the cone with top angle $\sim 90^{\circ}$. The axis of the cone was declined by $22.5^{\circ}$ from the orbital plane and directed $45^{\circ}$ upward from the Earth. The initial ion velocity corresponded to a bulk kinetic energy of $\sim 200 \mathrm{eV}$ and the total ion current could reach $2 \mathrm{~A}$.

The data presented in this paper have been obtained by the MPS/PPS low-energy and the DOK high-energy spectrometers on the MAGION-3 satellite. The MPS/PPS subsatellite spectrometer consists of two devices with independent sensor electronics and a common data processing unit. The electrons in both directions along the subsatellite main axis are registered by the cylindrical electrostatic analyzers of the small plasma spectrometer (MPS). The six toroidal analyzers of the PPS device (wide-angle plasma spectrometer) create a fan in the plane parallel to the main satellite's axis. This axis is oriented along the magnetic field by a system of magnetic orientation. Due to insufficient damping, the axis is swinging around the magnetic field line and in some cases the angle between the satellite's axis and the magnetic field vector can reach $70^{\circ}$. The energy range of both spectrometers is $0.1 \div 5 \mathrm{keV}$ or $0.2 \div 20 \mathrm{keV}$, divided into 16 steps in the logarithmic scale. The maximum time resolution is $0.8 \mathrm{~s}$ per spectrum. A detailed description can be found in Němeček et al. (1994).

The DOK spectrometer (Kudela et al., 1992) is designed for the registration of small charged particle flows in the energy range of $20 \mathrm{keV}$ to $1300 \mathrm{keV}$, which is divided into 8 energy channels separately for ions and electrons. Two pairs of silicon detectors parallel and perpendicular to the subsatellite's main axis are used. One detector is complemented by a magnetic filter for electron separation (proton detector). A foil is placed in front of the electron part to suppress the influence of the heavier particles and its metal coating protects this electron detector from the registration of the UV radiation.

A more complex set for plasma and charged particle investigations was placed on board the main satellite. The ion and electron distributions were measured in 24 directions simultaneously in the energy range from 0.05 to $30 \mathrm{keV}$ and the parameters of the cold plasma component were measured with Langmuir probes. A more detailed description of these facilities can be found in Němeček et al. (1997).

\section{Experimental results}

Field-aligned electron flows are a common feature of the magnetospheric auroral region and they are often observed by low orbiting satellites. However, the MAGION-3 satel- 

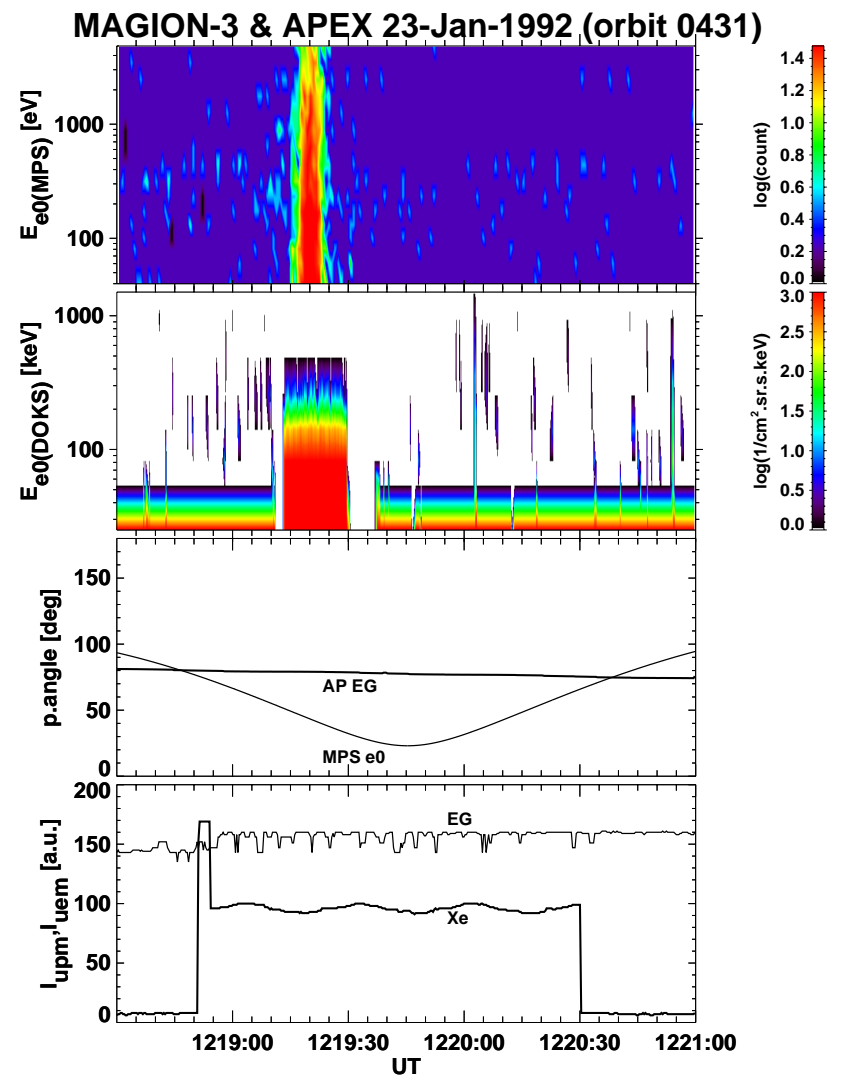

Fig. 1. Parameters of the electron burst observed during orbit No.

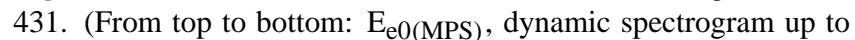
$5 \mathrm{keV}$ measured in direction parallel to the satellite axis by the MPS

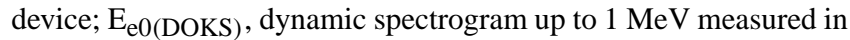
the same direction by the DOK device; p.angle, the pitch angle of both aforementioned channels (MPS e0) and pitch angle of the electron gun on board the IK-25 satellite (AP EG); $\mathrm{I}_{\text {upm }}, \mathrm{I}_{\mathrm{uem}}$, monitors of electron (EG) and ion (Xe) guns on board IK-25 satellite.

lite has observed about 20 strong field-aligned electron bursts well equatorward of the auroral oval $\left(25^{\circ} \div 50^{\circ}\right.$ of geomagnetic latitude). The dynamic energy spectrogram of one of these bursts is shown in Fig. 1. The data in the top panel have been collected by the channel of the MPS device oriented along the satellite axis during orbit No. 431. The burst is strongly limited in time and the energy of the observed electrons exceeds the upper limit of the measuring range $(5 \mathrm{keV}$ in this particular case). Using the data of the parallel channel of the DOK device looking in the same direction, the energy range can be extended to above $1 \mathrm{MeV}$, as it is shown in the second panel of Fig. 1. The duration of the event is longer and the energy of electrons reaches a few hundreds of $\mathrm{keV}$ in the DOK observations. A striking feature of the observed burst is a rectangular shape of the dynamic spectrogram. The counts appear simultaneously on all energies and remain constant throughout the event.

The MPS and DOK devices differ in angular apertures, which are $\pm 3^{\circ}$ and $\pm 15^{\circ}$, respectively. Providing that the real duration of the event is that observed by the DOK, the

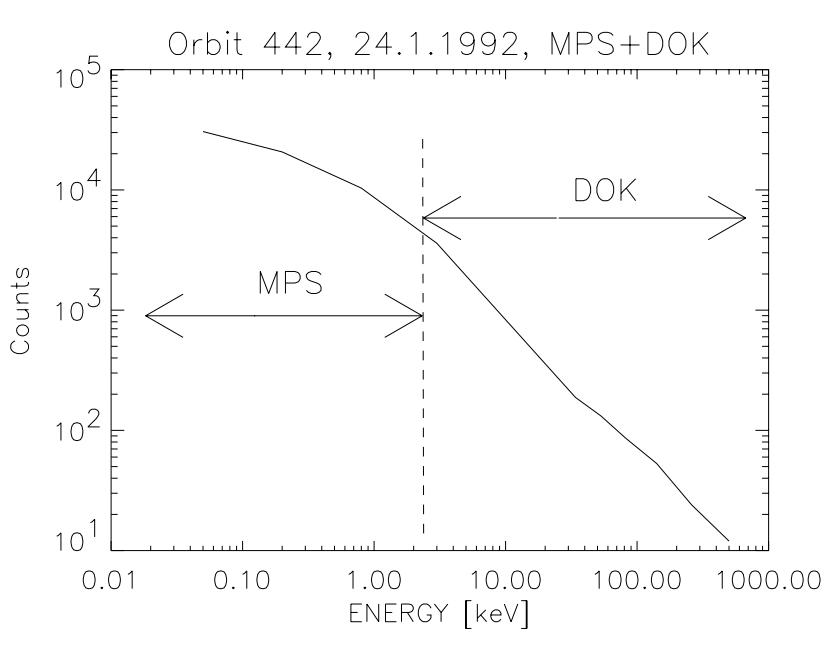

Fig. 2. Energy spectrum of the electron burst as a result from the data of two spectrometers, MPS and DOK.

MPS observation can be explained by the narrow (less than $10^{\circ}$ ) pitch-angular distribution of the burst electrons. This explanation is consistent with the changes in the channel pitch angle, which is plotted in the third panel of the figure (MPS e0 line).

Observed bursts are always registered by one channel of the MPS or PPS devices only. Due to the spacecraft swinging, the pitch angle of the particular channel changes quickly in time and the counts sometimes switch to another channel with the appropriate direction during one event. Under the presumption that the temporal duration of observed fluxes is significantly longer than that seen by the MPS device (this fact will be discussed later), it can be concluded that the angular width of the observed flow is less than $10^{\circ}$ but is centered at different angles with respect to the magnetic field direction. The indirect confirmation of this presumption can be done by the construction of the energy spectrum of the electron flow in the whole energy range covered by the measuring range of both devices. The resulting energy spectrum is depicted in Fig. 2. The slope of the spectrum is the same in both subranges and it can be supposed that both devices registered electrons of the same origin. It should be noted that the construction of the spectrum in Fig. 2 is rather artificial. The geometric factor of the MPS spectrometer is $7 \times 10^{-5} \mathrm{~cm}^{2} . \mathrm{sr}$ $10^{-2} \mathrm{~cm}^{2}$.sr for the DOK device; the pitch angle distribution of the observed flow is unknown. Nevertheless, we can suppose that the angular width of the flow is wider than the aperture of the MPS spectrometer but narrower than that of the DOK sensor. For these reasons, it is difficult to determine the relative calibration coefficient. It should be less than the ratio of the geometrical factors but greater than the ratio of the entrance areas. We have chosen a value of 450 which fullfills the requirements mentioned above and which gives a smooth energy spectrum for the event depicted in Fig. 2. Using this value, the plotted energy spectra of other observed events do not exhibit substantial discontinuities.

It should be noted that during the MAGION-3 registration 
of the electron burst, the active experiment that consisted of the simultaneous emissions of $8 \mathrm{keV}$ electrons and $\mathrm{Xe}$ ions was carried out on board main satellite. The satellites were separated by $\sim 450 \mathrm{~km}$ and the separation vector was nearly perpendicular to the magnetic field direction. The monitors of the ion plasma releaser and the electron gun currents are shown in the bottom panel of Fig. 1. The electrons were emitted continuously during the whole depicted interval and the ion current switching does not correspond to the appearance of the electron burst at the MAGION-3 position. The pitch angle of an electron gun is shown in third panel of Fig. 1 by the AP EG line.

The events similar to that depicted in Fig. 1 were observed by MAGION-3 during the period from the beginning of January to the end of February 1992, but only those fulfilling the following two criteria have been chosen for further consideration:

- The energy flux measured by the MPS device exceeds $3 \times 10^{5}\left[\mathrm{~cm}^{2} \text {.sr.s.keV }\right]^{-1}$;

- The event is located equatorward of the region of the auroral precipitation (INL $<55^{\circ}$ in general);

- The event was recorded by the MPS device because the acceptance angle of DOK was too broad to determine the pitch angle of the burst observed by DOK only;

The coordinates and other important parameters of the events chosen are listed in Table 1 (where $\alpha$ is the pitch angle of the measured bursts, $T_{d}$ and $\Delta$ INL are the distance (in seconds and along the orbit) and angular separation between both spacecraft, $K_{P}$ and $D_{S T}$ are geomagnetic indexes, MLT is the magnetic local time, INL is the invariant latitude, ALT is the satellite's altitude, activity time is the period of active operations on board the main satellite, and D in the last column stands for the events observed by both MPS and DOK spectrometers). It should be noted that the DOK device was switched off during all events observed by MPS only.

The data in Table 1 exhibit some features that should be pointed out:

- All events have been registered near the local noon;

- The distance between two spacecraft of the APEX project does not exceed $80 \mathrm{~s} \mathrm{(or} \sim 550 \mathrm{~km}$ in space) for all events listed in Table 1, although the maximum separation of the satellites was $\sim 2000 \mathrm{~km}$ and active experiments were performed in the whole range of distances;

- The magnetic local time (MLT) of the observed events decreases with time (or with the number of orbit);

- The satellite orbit lies in the plane defined by the magnetic field line. In other words, the satellite moves with MLT $=$ constant. It means that both APEX satellites are separated only latitudinally, not longitudinally;

- The pitch angle of burst particles increases with the increasing geomagnetic latitude as can be seen in Fig. 3,

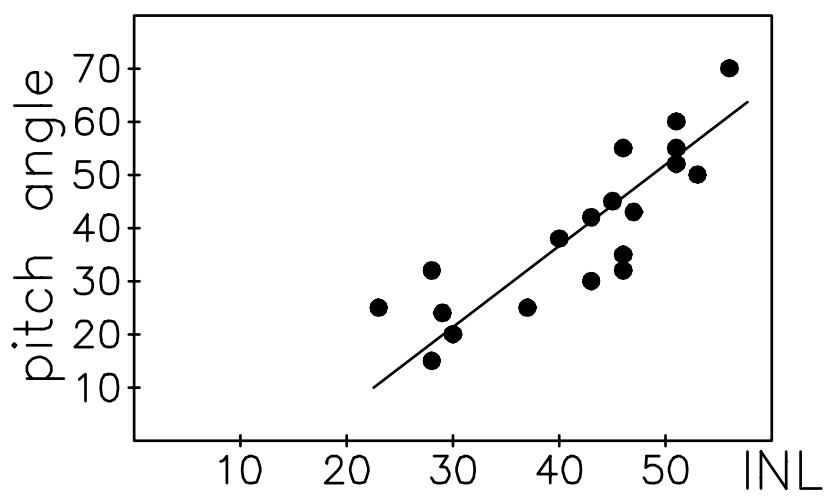

Fig. 3. The latitudinal dependence of the burst pitch angle.

where the pitch angle is shown as a function of INL. The heavy line shows the least square fit of measurements.

The localization of the burst observation to the altitudes from 800 to $1500 \mathrm{~km}$ is probably a consequence of the local time favourable for the observation, the satellite orbit, and other parameters. The pitch angles are lying below $90^{\circ}$. Since all observations were carried out in the Northern Hemisphere, the electrons are precipitating toward the atmosphere.

Other correlations, which can be deduced from Table 1, are probably caused by limitations of the experiment itself. Our data have been directly transmitted to the receiving station located near Prague, and thus, the measurements were carried out only if the satellites were in the direct visibility from this station. Due to this arrangement, although the UT and MLT are independent quantities in principle, they cannot differ substantially in our experiment. Nevertheless, the orbit geometry allows us to receive the data from three consecutive orbits that differ by $\sim 4$ hours of MLT.

The short duration of the electron burst, as shown in Fig. 1 is not typical. The bursts can last for a few minutes or they can be observed repeatedly. An example of multiple observations is plotted in Fig. 4. The MPS energy spectra in the top panel show three short electron bursts. Three longer intervals of high-energy electrons presence can be identified in the DOK measurements (middle panel) as well. The pitch angle of the MPS detector is plotted in the last panel with a dashed line and the range of pitch angles covered by the DOK sensor is indicated by two thin lines. We suppose that a narrow pitch-angle distribution of registered electrons is centered around the MPS observations and that it evolves in time. The temporal evolution of the pitch angle is fitted by the straight line in the last panel of Fig. 4; it is qualitatively consistent with the latitudinal dependence of the bursts pitch angle plotted in Fig. 3.

The assumed temporal (or latitudinal) dependence can explain the relation between the MPS and DOK observations because the DOK observes high-energy electrons from 12:35:40 to $12: 36: 35 \mathrm{UT}$, from $12: 38: 10$ to $12: 39: 10 \mathrm{UT}$, and again from 12:40:00 to 12:40:50 UT, when the burst pitch angle lies inside its acceptance angle. On the other hand, the MPS registers the electrons only if their pitch 
Table 1. Conditions for the electron bursts

\begin{tabular}{|c|c|c|c|c|c|c|c|c|c|c|c|c|}
\hline orbit & date & UT & MLT & $\begin{array}{c}\text { INL } \\
\left.{ }^{\circ}\right]\end{array}$ & $\begin{array}{l}\text { ALT } \\
{[\mathrm{km}]}\end{array}$ & $\begin{array}{c}\alpha \\
{\left[{ }^{\circ}\right]}\end{array}$ & $\begin{array}{l}T_{d} \\
{[\mathrm{~s}]}\end{array}$ & $\begin{array}{c}\Delta \mathrm{INL} \\
{\left[{ }^{\circ}\right]}\end{array}$ & $K_{P}$ & $\begin{array}{l}D_{S T} \\
{[\mathrm{nT}]}\end{array}$ & $\begin{array}{c}\text { activity } \\
\text { time }\end{array}$ & $\mathrm{P}$ \\
\hline 325 & $14 / 01 / 92$ & $13: 26: 00$ & $15: 01$ & 28 & 810 & $15^{\circ}$ & -80 & +5.6 & 3 & -2 & $13: 21: 14-13: 30: 53$ & \\
\hline 349 & $16 / 01 / 92$ & $\begin{array}{l}14: 02: 40 \\
14: 06: 00\end{array}$ & $\begin{array}{l}14: 50 \\
14: 45\end{array}$ & $\begin{array}{l}40 \\
26\end{array}$ & $\begin{array}{l}1402 \\
1406\end{array}$ & $\begin{array}{l}38^{\circ} \\
32^{\circ}\end{array}$ & $\begin{array}{l}-71 \\
-71\end{array}$ & +5 & 3 & -27 & $14: 03: 06-14: 08: 32$ & \\
\hline 360 & $17 / 01 / 92$ & $12: 20: 40$ & $14: 47$ & 43 & 700 & $42^{\circ}$ & -64 & +4.1 & 2 & -16 & $12: 16: 10-12: 25: 52$ & \\
\hline 431 & $23 / 01 / 92$ & $12: 19: 20$ & $14: 12$ & 47 & 830 & $43^{\circ}$ & -33 & +2 & 1 & 2 & $12: 19: 06-12: 24: 32$ & $\mathrm{D}$ \\
\hline 442 & $24 / 01 / 92$ & $10: 36: 00$ & $14: 02$ & 53 & 800 & $50^{\circ}$ & -27 & +1.6 & 1 & 10 & & $\mathrm{D}$ \\
\hline 455 & $25 / 01 / 92$ & $\begin{array}{l}13: 00: 20 \\
13: 00: 30 \\
13: 04: 30\end{array}$ & $\begin{array}{l}13: 56 \\
13: 56 \\
13: 50\end{array}$ & $\begin{array}{l}46 \\
45 \\
30\end{array}$ & $\begin{array}{c}930 \\
930 \\
1200\end{array}$ & $\begin{array}{l}55^{\circ} \\
45^{\circ} \\
20^{\circ}\end{array}$ & $\begin{array}{l}-20 \\
-20 \\
-20\end{array}$ & +1.2 & 1 & 1 & & $\mathrm{D}$ \\
\hline 466 & $26 / 01 / 92$ & $11: 16: 40$ & $13: 53$ & 51 & 850 & $55^{\circ}$ & -13 & +0.8 & 1 & 15 & $11: 11: 06-11: 14: 24$ & $\mathrm{D}$ \\
\hline 479 & $27 / 01 / 92$ & $\begin{array}{l}13: 43: 20 \\
13: 47: 30\end{array}$ & $\begin{array}{l}13: 37 \\
13: 30\end{array}$ & $\begin{array}{l}37 \\
23\end{array}$ & $\begin{array}{l}1130 \\
1400\end{array}$ & $\begin{array}{l}25^{\circ} \\
25^{\circ}\end{array}$ & $\begin{array}{l}-15 \\
-15\end{array}$ & +0.8 & 2 & -30 & & \\
\hline 514 & $30 / 01 / 92$ & $\begin{array}{l}12: 38: 28 \\
12: 38: 40 \\
12: 40: 38\end{array}$ & $\begin{array}{l}13: 27 \\
13: 27 \\
13: 23\end{array}$ & $\begin{array}{l}51 \\
51 \\
43\end{array}$ & $\begin{array}{l}1010 \\
1010 \\
1150\end{array}$ & $\begin{array}{l}52^{\circ} \\
60^{\circ} \\
30^{\circ}\end{array}$ & $\begin{array}{l}+8 \\
+8 \\
+8\end{array}$ & -0.4 & 3 & -24 & $12: 39: 54-12: 45: 20$ & \\
\hline 538 & $01 / 02 / 92$ & $13: 25: 20$ & 13:01 & 29 & 1500 & $24^{\circ}$ & +10 & -0.6 & $3+$ & -30 & $13: 20: 58-13: 26: 24$ & $\mathrm{D}$ \\
\hline 561 & $03 / 02 / 92$ & $11: 56: 32$ & 13:07 & 56 & 1070 & $70^{\circ}$ & +50 & -2.6 & 5 & -110 & 11:59:54-12:03:12 & $\mathrm{D}$ \\
\hline 584 & $05 / 02 / 92$ & $\begin{array}{l}10: 38: 00 \\
10: 38: 20 \\
10: 38: 50\end{array}$ & $\begin{array}{l}12: 46 \\
12: 46 \\
12: 46\end{array}$ & $\begin{array}{l}46 \\
46 \\
46\end{array}$ & $\begin{array}{l}1315 \\
1315 \\
1315\end{array}$ & $\begin{array}{l}35^{\circ} \\
32^{\circ} \\
32^{\circ}\end{array}$ & $\begin{array}{l}+37 \\
+37 \\
+37\end{array}$ & -2 & $2+$ & -60 & 10:38:02-10:43:28 & \\
\hline
\end{tabular}

angle is equal to the detector pitch angle, at 12:38:30 UT and at 12:40:35 UT. In this interpretation, the gradual decreasing/increasing of the counts around 12:39-12:40 UT is caused by the scanning of the energy-pitch angle distribution of the observed electrons. It means that the range of pitch angles of the particles inside the burst increases with the decrease of their total energy. This behaviour suggests that the bursts were created from the low-temperature plasma by some acceleration process which is effective only in one direction with respect to the magnetic field (e.g. field-aligned potential drop) and the spread of the pitch angles is caused by the original thermal velocity distribution.

The abrupt changes in the DOK spectra at 12:38:10, 12:40:47, and (probably) at 12:36:40 UT can be caused by the exit/enter of the satellite from/to the region occupied by the high-energy electrons.

The electron bursts reported in the previous section were observed equatorward of the auroral region and below the zone of trapped radiation. In this particular case, the electron burst was observed from 12:35:00 to 12:41:20 UT, i.e. 380 s. This duration correspond to a distance of about $3800 \mathrm{~km}$, but a perpendicular dimension of the burst could be different. The bursts were observed exceptionally by the MAGION3 satellite. This satellite was followed by the IK-25 satellite which was equipped by much more sophisticated and com- plex electron spectrometers and separated by tens or hundreds of kilometers. However, this satellite did not register any of these bursts.

An example of such simultaneous observations of both satellites is depicted in Fig. 5. The top two panels show MAGION-3 measurements of the electron distribution. The IK-25 electron energy distribution is shown in the last panel for the pitch angle identical with the pitch angle of the electrons observed by MAGION-3. It can be noted that the counts in the last panel are comparable to the noise level. The spacecraft separation was $\sim 470 \mathrm{~km}$ in this particular case but we have carefully analyzed January and February 1992 data and we have not found any observations similar to that of MAGION-3 in the IK-25 measurements. The minimum separation of MAGION-3 and IK-25 spacecraft during burst observation was $64 \mathrm{~km}$. It means that the real cross section of the burst would be even smaller and the long duration of the burst observation in Fig. 4 is caused by the simultaneous motion of the burst and spacecraft. On the other hand, the largest separation of the spacecraft during burst observations was about $550 \mathrm{~km}$ (first row of Table 1). We think that the principal finding following from Table 1 is that the bursts were observed on the orbits with MLT $=$ constant. Such orbits were in direct visibility from the receiving station only during the period covered by Table 1 and the spacecraft sep- 


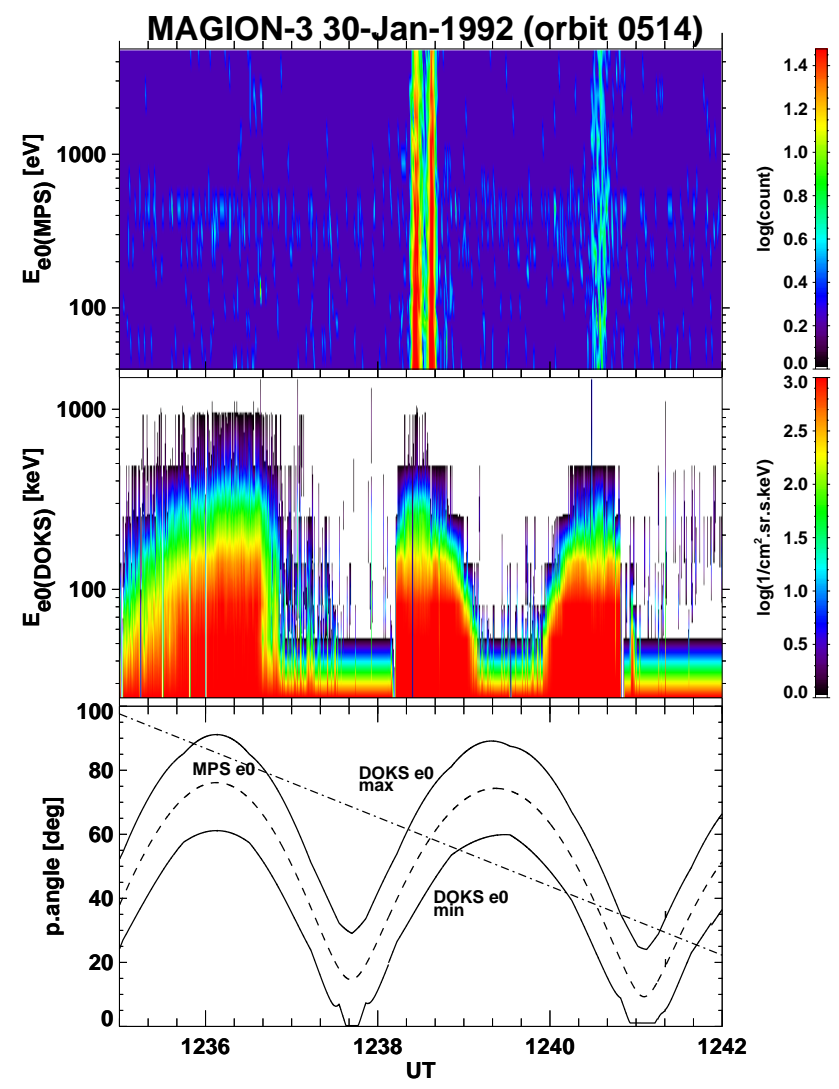

Fig. 4. Multiple observations of the electron burst (orbit No. 514). From top to bottom: $\mathrm{E}_{\mathrm{e} 0 \text { (MPS) }}$, dynamic spectrogram registered

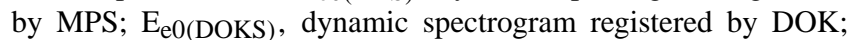
p.angle, pitch angle of MPS (MPS e0 and dashed line), the range of pitch angles covered by DOK lies between two thin curves (DOKS e0 min, DOKS e0 max), dotted-dashed line shows approximate change of burst pitch angle with the time.

aration did not exceed the aforementioned distance during this period. For this reason, we cannot say if this distance represents an upper limit for observations.

\section{Discussion}

The features which can be derived from our observations can be summarized as follows:

1. The bursts were recorded on the orbits with MLT = constant;

2. The burst cross section is spatially limited to several tens of kilometers in the direction of the s/c orbit;

3. The bursts move together with the spacecraft;

4. The energy of burst particles extends to several hundreds of $\mathrm{keV}$;

5. The burst pitch angle increases with latitude.

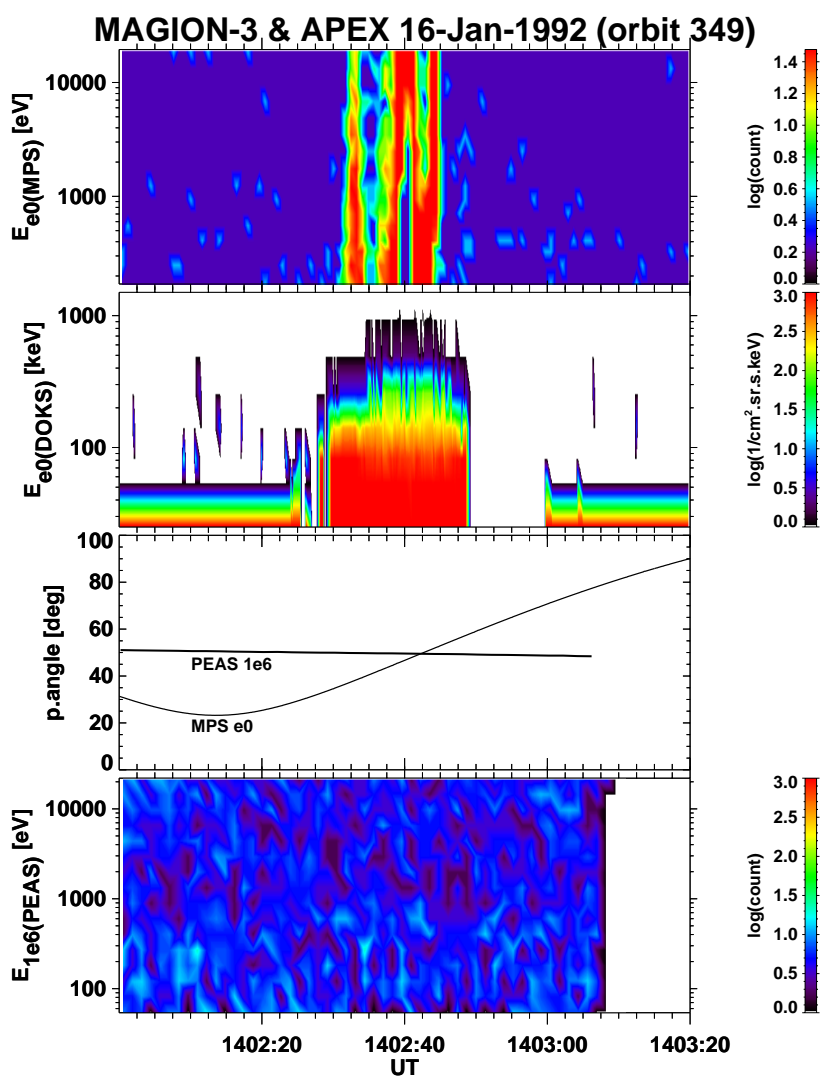

Fig. 5. Simultaneous observations of the electron energy spectra in two shortly separated points. Two top panels show energy spec-

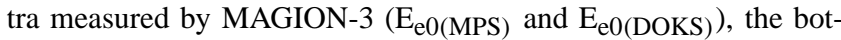
tom panel shows the spectrum measured by the PEAS spectrometer $\left(\mathrm{E}_{1 \mathrm{e} 6(\mathrm{PEAS})}\right)$ onboard the IK-25 satellite. The separation between both satellites was $\sim 450 \mathrm{~km}$.

These facts, together with the absence of similar observations by other spacecraft orbiting in this region, lead us to a conclusion that the reported electron bursts are a product of active experiments on board the IK-25 satellite. There are two possible sources of the observed electrons: (1) the electrons emitted from the main satellite or the electrons from ambient plasma, both accelerated to the observed energies, and (2) the ring current electrons released by some mechanism induced by the particles emitted from the IK-25 satellite.

If we follow the idea of the main satellite being the source of the observed particles, we should discuss the following questions:

1. Is the beam-plasma interaction able to accelerate the electrons to the observed energies?

2. Which way do the observed electrons move across the magnetic field lines?

3. Why are the electrons observed only in part of the active experiments?

The answer to the first question is probably the most difficult and for this reason we will discuss it as the last point. 
For the discussion of the other questions, we will suppose that the answers are positive. We can divide the motion of the guiding centers of observed electrons into two parts: the latitudinal and the longitudinal component (in the geomagnetic coordinate system). For the sake of simplicity, we will consider only the dipole magnetic field of the Earth.

In this field, $\operatorname{grad} \boldsymbol{B}$ has no longitudinal component and thus, the velocity of the $\operatorname{grad} \boldsymbol{B}$ drift has longitudinal direction; its value can be estimated roughly from the following formula:

$v_{\text {grad } B} \sim \pm v_{\perp} r_{L} \frac{1}{R}$

where $v_{\perp}$ is the velocity of the particle perpendicular to the magnetic field, $r_{L}$ is the Larmor radius, and $R$ is the distance from the Earth's centre. The $\mathbf{g r a d} \boldsymbol{B}$ drift is a quadratic function of the particle velocity and can lead to longitudinal dispersion of the observed beam. However, the velocity and pitch angle of the particles taken from our experiment lead to values of the order of units or tens of $\mathrm{m} \cdot \mathrm{s}^{-1}$ and thus, the process of the gradual dispersion is very slow.

On the other hand, the $\operatorname{grad} \boldsymbol{B}$ drift velocity depends on the sign of the particle charge and leads to charge separation and creation of electric field. This field should be perpendicular to the magnetic field because the parallel component is immediately compensated due to highly anisotropic conductivity. If we suppose a parallel beam, the spectrum in Fig. 2 corresponds to the density $n_{e} \sim 30 \mathrm{~m}^{-3}$. In one-dimensional approximation, which can be used for the estimation of the electric field inside the beam, the electric field, $E$ is a linear function of the distance from the beam centre, $x$ :

$$
E=\frac{x n_{e} e}{\varepsilon_{o}} .
$$

If the dimension of the beam in the electric field direction is of the order of kilometers, the intensity of the electric field can reach tens of $\mathrm{mV} / \mathrm{m}$. Then the real value would be probably lower, since the shielding effect was neglected in this consideration. On the other hand, the dimensions of bursts would be significantly lower and the densities significantly higher in the vicinity of the emitting satellite and thus, the electric field would be larger there. The emitting satellite moves roughly in the latitudinal direction and, in simple approximation, the product of the beam-plasma interaction fills a part of the meridional plane. The electric field has only the longitudinal component in this geometry and causes the $\boldsymbol{E} \times \boldsymbol{B}$ drift with the velocity $v_{E \times B}$ :

$v_{E \times B}=\frac{E}{B}$

which is directed latitudinally.

Under the conditions mentioned above, the drift velocity can reach $10^{2} \div 10^{3} \mathrm{~m} \cdot \mathrm{s}^{-1}$. This velocity is less than the spacecraft velocity, but according to Němeček et al. (1997), the intensity of the electric field in the vicinity of the emitting spacecraft can reach a value of $100 \mathrm{~V} / \mathrm{m}$. The $\boldsymbol{E} \times \boldsymbol{B}$ drift velocity corresponding to this value is of the order of
$10^{7} \mathrm{~m} \cdot \mathrm{s}^{-1}$. The mean electric field needed for the particles emitted from one spacecraft to be seen by the other is about $300 \mathrm{mV} / \mathrm{m}$. We think that the mean field of such value can be expected.

The effects described above are stronger when the satellite moves in the plane defined by the magnetic field line because the active agents emitted from the main spacecraft and the products of the beam-plasma interactions remain in this plane. If the orbital plane is not identical with the meridional plane, the emitted particles are spread into a huge volume and their density would be too small to cause a detectable effect. This is consistent with our observations, since all electron bursts were observed only when the orbital plane of the satellites coincided with the plane defined by the magnetic meridian (under conditions schematically shown in Fig. 6). A depicted geometry refers to the APEX No. 442 orbit and to the time of the burst observation. This figure represents a cut along the geomagnetic meridian which contains a part of the APEX orbit. The positions of both satellites are marked, the subsatellite is located ahead (equatorward) of the main satellite, the direction of the satellite's motion is indicated by arrow. One can clearly see that the products of the beamparticle interaction from the disturbed area can reach the subsatellite due to the $\boldsymbol{E} \times \boldsymbol{B}$ drift velocity, which is perpendicular to the magnetic field. This mechanism is able to explain the periodicity of the electron burst observations if the temporal evolution of the satellite orbit in geomagnetic coordinates is taken into account. If we suppose that part of the satellite's orbit defined by a range of latitudes lies in the plane of the magnetic meridian, the next orbit will be out of such a plane due to the Earth's rotation. This situation will repeat itself the next day at the same time and MLT, providing that $24 \mathrm{~h}$ can be divided by the orbital period with zero reminder. It is not exactly true for the APEX orbit and thus, there is a shift of $\sim 6$ minutes per day. This shift corresponds roughly with the change in the longitudinal position of electron bursts in the observed data, as follows from Table 1 . The deviation from the predicted position of the observation can be caused either by the non-zero width of the electron beam or by the changes of the magnetic field during the disturbed geomagnetic periods (see $K_{P}$ and $D_{S T}$ indices in Table 1). Moreover, the grad B drift, which we neglected in our simplified considerations, causes a systematic declination of the emitted particles from the meridional plane.

The last problem is whether or not the disturbances induced by the electron and/or ion gun are able to accelerate particles to the observed energies. The guns supply the space around satellite by $\sim 800 \mathrm{~J} \doteq 10^{22} \mathrm{eV}$ of free energy each second. If we assume a density of accelerated particles $\sim 30 \mathrm{~m}^{-3}$ and a mean energy of $\sim 1 \mathrm{keV}$, the energy density carried by these particles is $\sim 3 \times 10^{4} \mathrm{eV} . \mathrm{m}^{-3}$. The volume of the region that contains accelerated particles is probably less than $10^{15} \mathrm{~m}^{3}$ and thus, these particles carry less than one percent of the electron gun energy.

A number of theories for the electron acceleration in the magnetosphere have been proposed (see Lundin, 1991 for a review) - magnetic mirroring, double layers, Alfvén or lower 


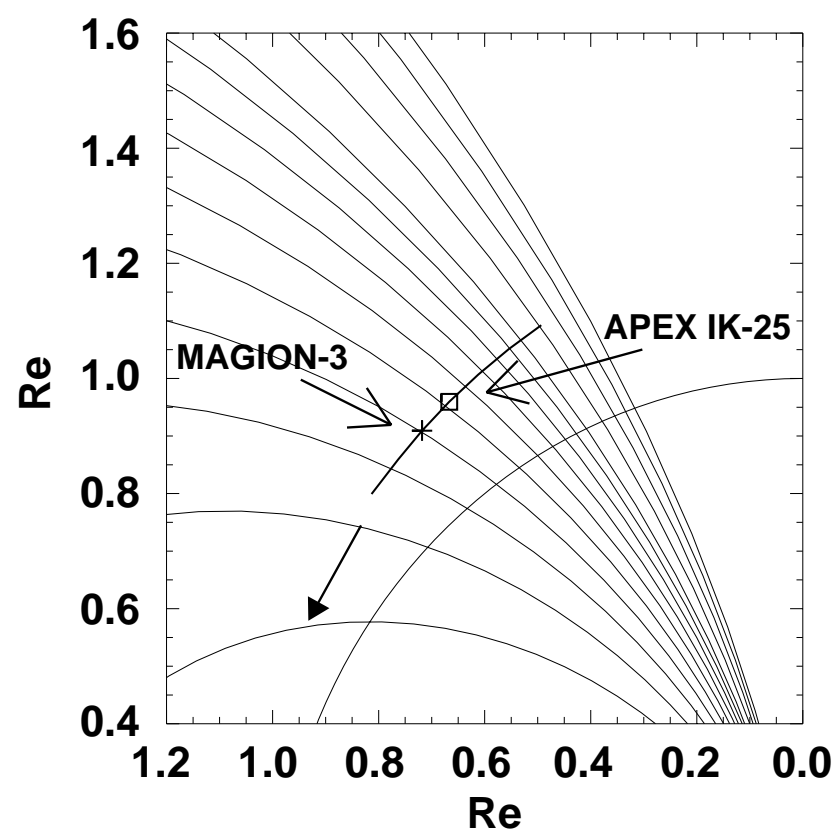

Fig. 6. Schematic plot of satellite positions during electron burst observations.

hybrid waves, field-aligned potential drop, etc. It is a question if some of these mechanisms or a combination of two or more of them are able to accelerate particles to the observed energies under conditions of the active experiment.

The particles inside the zone disturbed by the electron gun move in a highly inhomogeneous nonstationary electric field which leads to their acceleration (Němeček et al., 1997). The acceleration is a function of the particle pitch angle but this function is not too sharp, as follows from our observations. On the other hand, observations in Němeček et al. (1997) have been made by the particle energy spectrometer placed on the emitting satellite and thus, they cannot show fully developed disturbances. The Larmor radius of particles emitted from the gun is $\sim 10 \mathrm{~m}$ and the satellite moves across the magnetic field lines with a velocity of $\sim 5 \times 10^{3} \mathrm{~m} . \mathrm{s}^{-1}$, so that it leaves the disturbed region in a few ms.

Computer simulations show that part of the electrons emitted from the gun can be accelerated to energies which highly exceed the gun energy (Pritchett, 1990). Unfortunately, the conditions of these simulations are too simplified (uniform magnetic field, non-moving source, unrealistic ratio between the electron and ion masses, etc.) to describe the real situation.

An alternative source of the observed high-energy electrons can be the zone of the trapped radiation. This zone produces spontaneously microbursts of high-energy electrons in the dayside sector, but in higher geomagnetic latitudes than the bursts reported in this study. The mechanism of this anomalous precipitation is generally unknown but if we suppose that it can be amplified by the process of the beamplasma interaction, we can probably expect the bursts of a longer duration and in lower latitudes.

\section{Conclusion}

The paper presents a study of the electron bursts observed by the MAGION-3 satellite in low geomagnetic latitudes during the first months of the APEX experiment. The main results of the study are:

1. The basic parameters of the observed electron bursts are:

- burst energies up to several keV;

- narrow pitch-angle distributions (less than $10^{\circ}$ );

- none or negligible temporal dispersion in whole energy range.

2. Two possible sources have been suggested:

- an energization of the electrons emitted from the IK-25 satellite or electrons from the ambient plasma due to the beam-plasma interaction;

- an anomalous precipitation of the ring current particles induced by the beam-plasma interaction.

3. The sources are effective only if the emitting satellite moves in the meridional plane.

The temporal and spatial dependencies present in the MAGION-3 observations have been explained but the study leaves as an open question the problem of the acceleration of electrons to observed energies. This problem is rather complex and cannot be solved without an appropriate computer model of the beam-plasma interaction that involves all peculiarities of the particular experiment. Such model would explain the narrow pitch angle distribution of the observed electrons, the slope of the energy distribution, and the dependence of the burst pitch angle on the latitude of the observation.

Acknowledgement. The authors would like to thank K. Kudela for the DOK instrument data and V. S. Dokukin for information on active APEX regimes. The present work was carried out as a part of the Research project, MSM 113200004 and partly supported by the Czech Grant Agency under Contract No. 202/99/M004.

Topical Editor G. Chanteur thanks four referees for their help in evaluating this paper.

\section{References}

Anderson, K. A. and Milton, D. W.: Balloon observations of X-rays in the auroral zone, 3, High time resolution studies, J. Geophys. Res., 69, 4457-4466, 1964.

Arnoldy, R. L. and Winckler, J. R. : The hot plasma environment and floating potentials of an electron-beam-emitting rocket in the ionosphere, J. Geophys. Res., 86, 575-584, 1981.

Bering, E. A., Benbrook, J. R., Leverenz, H., Roeder, J. L., Stansbery, E. G., and Sheldon, W. R.: Longitudinal differences in electron precipitation near $L=4$, J. Geophys. Res., 93, $11385-$ $11403,1988$. 
Boehm, M. H., Paschmann, G., Clemmons, J., Haerendel, G., Eliasson, L., and Lundin, R.: Freja observations of narrow inverted-V electron precipitation by the two-dimensional electron spectrometer, Geophys. Res. Lett., 21, 1895-1898, 1994.

Büchner, J. and Zelenyi, L. M.: Regular and chaotic charged particle motion in magnetic taillike field reversals, 1 , Basic theory of trapped motion, J. Geophys. Res., 94, 11 821-11 842, 1989.

Clemmons, J. H., Boehm, M. H., Paschmann, G. E., and Haerendel, G.: Signatures of energy-time dispersed electron fluxes measured by FREJA, Geophys. Res. Lett., 21, 1899-1902, 1994.

Dokukin, V. S.: The APEX project scientific facility orbital complex, in Project APEX. Scientific Purposes, simulation, technology and equipment of experiment, Nauka, Moscow, 16-29, 1992.

Duprat, G. R., Whalen, B. A., McNamara, A. G., and Bernstein, W.: Measurements of the stability of energetic electron beams in the ionosphere, J. Geophys. Res., 88, 3095-3102, 1983.

Haerendel, G., Frey, H. U., Bauer, O. H., Rieger, E., Clemmons, J., Boehm, M. H., Wallis, D. D., and Lühr, H.: Inverted-V events simultaneously observed with Freja satellite and from the ground, Geophys. Res. Lett., 21, 1891-1894, 1994.

Hardy, D. A., Oberhard, R. M., and Burke, W. J.: Observations of electron beam propagation perpendicular to the Earth's magnetic field during the TSS 1 mission, J. Geophys. Res., 100, $21523-$ $21534,1995$.

Hastings, D. E.: A review of plasma interactions with spacecraft in low Earth orbit, J. Geophys. Res., 100, 14 457-14 483, 1995.

Imhof, W. L.: Fine resolution measurements of L-dependent energy threshold for isotropy at the trapping boundary, J. Geophys. Res., 93, 9743-9752, 1988.

Imhof, W. L., Voss, H. D., Reagan, J. B., Datlowe, D. W., Gaines, E. E., Mobilia, J., and Evans, D. S.: Relativistic electron and energetic ion precipitation spikes near the plasmapause, J. Geophys. Res., 91, 3077-3088, 1986.

Imhof, W. L., Voss, H. D., Mobilia, J., Walt, M., Inan, U. S., and Carpenter, D. L.: Characteristics of short-duration electron precipitation bursts and their relationship with VLF wave activity, J. Geophys. Res., 94, 10 079-10 093, 1989.

Imhof, W. L., Voss, H. D., Mobilia, J., Datlowe, D. W., and Gaines, E. E.: The precipitation of relatiristic electrons near the trapping boundary, J. Geophys. Res., 96, 15619-5629, 1991.

Imhof, W. L., Voss, H. D., Mobilia, J., Datlowe, D. W., Gaines, E. E., and McGlennon, J. P.: Relativistic Electron Microbursts, J. Geophys. Res., 97, 13 829-13 837, 1992.

Imhof, W. L.,Chenette, D. L., and Gaines, E. E.: Characteristics of electrons at the trapping boundary of the radiation belt, J. Geophys. Res., 102, 95-104, 1997.

Jacobsen, T. A.: Observations of plasma heating effects in the ionosphere by a rocket borne electron accelerator, in: Artificial Particle Beams in Space Plasma Studies, (Ed) Grandal, B., New York, 1982.

Johnstone, A. D. and Winningham, J. D.: Satellite observation of suprathermal electron bursts, J. Geophys. Res., 87, 2321-2329, 1982.

Kudela, K., Matisin, J., Shiskaya, F. K., Akentieva, O. S., Romantsova, T. V., and Venkatesan, D.: Inner zone electron peaks observed by the Active satellite, J. Geophys. Res., 97, 8681$8683,1992$.
Lotko, W.: Diffusive acceleration of auroral primaries, J. Geophys. Res., 91, 191-203, 1986.

Lundin, R. and Eliasson, L.: Auroral energization processes, Ann. Geophysicae, 9, 202-223, 1991.

Maehlum, B. N., Grandal, B., Jacobsen, T. A., and Troim, J.: Polar 5 - An electron accelerator experiment within an aurora, Planet. Space Sci., 28, 279-289, 1980.

McFadden, J. P., Carlson, C. W., and Boehm, M. H.: Field-aligned precipitation at the edge of an arc, J. Geophys. Res., 91, 17231730, 1986.

McFadden, J. P., Carlson, C. W., and Boehm, M. H.: Structure of an energetic narrow discrete arc, J. Geophys. Res., 95, 6533-6547, 1990.

Němeček, Z., Šafránková, J., Omar, A., and Polášek, C.: Short time high energy electron fluxes in day-side magnetosphere, Adv. Space Res., 17, 10, 35-38, 1996.

Němeček, Z., Šafránková, J., Santolík, O., Šimunek, J., Šmilauer, J., Fischer, S., Shutte, N. M., Vedenov, N. Y., Teltsov, M. V., and Marjin, B. V.: Two point measurement of energy distribution relaxation: APEX project, in: Solar Terrestrial Energy Program, (Ed) Baker, D., 159, 1994.

Němeček, Z., Šafránková, J., Přech, L., Šimunek, J., Šmilauer, J., Gringauz, K. I., Shutte, N. M., Teltsov, M. V., Marjin, B. V., Ruzhin, Y. Y., and Dokukin, V. S.: Artificial electron and ion beam effects: APEX experiment, J. Geophys. Res., 102, A1, 2201-2211, 1997.

Oberhardt, M. R., Hardy, D. A., Thompson, D. C., Raitt, W. J., Melchioni, E., Bonifazi, C., and Gough, M. P.: Positive Spacecraft Charging as Measured by the Shuttle Potential and Return Electron Experiment, IEEE Transactions on Nuclear Science, 40, 1532-1541, 1993.

Oliven, M. N., Venkatesan, D., and McCracken, K. G.: Microburst phenomena, 2, Auroral-zone electrons, J. Geophys. Res., 73, 2345-2354, 1968.

Popielawska, B., Szalinska-Piechota, E., and Tsyganenko, N. A.: On the nonadiabatic particle scattering in the Earth's magnetotail current sheet, Planet. Space Sci., 33, 1433-1437, 1985.

Pritchett, P. L.: Spatial coherence during pulsed injection of electron beams, J. Geophys. Res., 95, $10671-10677,1990$.

Sagdeev, R. Z., Managadze, G. G., Mayorov, A. D., Lyakov, S. B., Martinson, A. A., Romanovsky, Y. A., Adeiskvily, T. G., Leonov, N. A., and Gagua, T. I.: Peculiarities of the environment disturbance during the electron beam injection from the rocket, Adv. Space Res., 1, 77-81, 1981.

Temerin, M., McFadden, J. P., Boehm, M., Carlson, C. W., and Lotko, W.: Production of flickering aurora and field-aligned electron flux by electromagnetic ion cyclotron waves, J. Geophys. Res., 91, 5769-5792, 1986.

Torbert, R. B. and Carlson, C. W.: Evidence for parallel electric field particle acceleration in the dayside auroral oval, J. Geophys. Res., 85, 2909-2914, 1980.

Winckler, J. R., Steffen, J. E., Malcolm, P. R., Erickson, K. N., Abe, Y., and Swanson, R. L.: Ion resonances and ELF wave production by an electron beam injected into the ionosphere: Echo 6, J. Geophys. Res., 89, 7565-7571, 1984.

Winningham, J. D., Stone, N. H., Gurgiolo, C. A., Wright, K. H., Frahm, R. A., and Bonifazi, C. A.: Suprathermal electrons observed on the TSS-1R satellite, Geophys. Res. Let., 25, 429-432, 1998. 